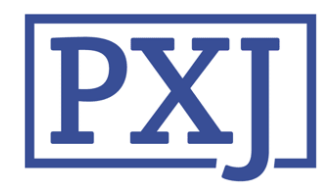

Patient Experience Journal

Volume 8

Issue 2 The Impact of Inequity \& Health

Disparities on the Human Experience

Article 12

2021

\title{
Building patient participation in quality of care through the healthcare stories project: A demonstration program in New York State HIV clinics
}

\author{
Abigail Baim-Lance \\ James J. Peters Veterans Health Administration and Icahn School of Medicine, Mount Sinai \\ Freda Coren \\ The University of Texas at Austin Dell Medical School \\ Margaret Brown \\ Hazel Lever \\ Massachusetts General Hospital, Department of Medicine \\ Daniel Tietz
}

See next page for additional authors

Follow this and additional works at: https://pxjournal.org/journal

Part of the Health and Medical Administration Commons, Health Policy Commons, Health Services Administration Commons, and the Health Services Research Commons

\section{Recommended Citation}

Baim-Lance A, Coren F, Brown M, Lever H, Tietz D, Agins B. Building patient participation in quality of care through the healthcare stories project: A demonstration program in New York State HIV clinics. Patient Experience Journal. 2021; 8(2):84-93. doi: 10.35680/2372-0247.1552.

This Case Study is brought to you for free and open access by Patient Experience Journal. It has been accepted for inclusion in Patient Experience Journal by an authorized editor of Patient Experience Journal. 


\section{Building patient participation in quality of care through the healthcare stories project: A demonstration program in New York State HIV clinics}

\section{Cover Page Footnote}

We would like to acknowledge each of the HCSP participating clinics' patients and staff who took part in the activities and the evaluation. We would also like to deeply thank Michele Canfield for her excellent design work on HCSP materials, and the NYS Department of Health statewide Consumer Advisory Committee for their active engagement with this initiative. This paper is dedicated to the memory of Humberto Cruz, former AIDS Institute Director, and champion of advancing coproduction with the PLWHA community. This article is associated with the Quality \& Clinical Excellence lens of The Beryl Institute Experience Framework (https://www.theberylinstitute.org/ExperienceFramework). You can access other resources related to this lens including additional PXJ articles here: http://bit.ly/PX_QualityClinExc

\section{Authors}

Abigail Baim-Lance, Freda Coren, Margaret Brown, Hazel Lever, Daniel Tietz, and Bruce Agins 


\author{
Building patient participation in quality of care through the healthcare \\ stories project: A demonstration program in New York State HIV clinics \\ Abigail Baim-Lance, James J. Peters Medical Center, Veterans Health Administration and Icahn School of Medicine at \\ MountSinai, abigail.baim-lance@va.gov \\ Freda Coren, The University of Texas at Austin Dell Medical School,freda.coren@gmail.com \\ Margaret Brown, margaret.k.brown@gmail.com \\ Hazel Lever, Massachusetts General Hospital, Department of Medicine, blever@partners.org \\ Daniel Tietz,dtiet266@gmail.com \\ Bruce Agins, University of California San Francisco, Institute for Global Health Sciences, bruce.agins@ucsf.edu
}

\begin{abstract}
There is growing recognition that patients should play a central role in defining, assessing, and improving the quality of healthcare, thereby enhancing patient experiences. Healthcare organizations struggle to meet these goals, which require becoming more patient-centered and patient-involved. The Healthcare Stories Project (HCSP), a demonstration program of the NYS Department of Health AIDS Institute, aimed to address this. HCSP comprises three, stepwise activities to: 1) Capture how patients define and experience 'quality of care' in the clinic; 2) Engage patients and providers as equal partners in understanding and improving the quality of care; and through partnerships, 3) Support the building of a coproduced healthcare system. After reviewing HCSP and its rollout in New York HIV outpatient settings, we describe a qualitative process evaluation, consisting of interviews at two time points with implementing organizations $(\mathrm{N}=12,11)$. Each activity offered an opportunity to share ideas and experiences of quality of care, generating concrete improvement project ideas. Activities strengthened patient involvement by engaging consumer advisory boards, and staff not traditionally involved in quality. While designed to be implemented with HIV patients, organizations implemented Activity Two and Three with broader populations. Organizations had the hardest time implementing Activity Three that focused on the coproduction concept, but they none the less applied and strengthened coproduced healthcare during Activities One and Two. Overall, HCSP is a promising model to advance patient-centered and patient-partnered quality of care, better understanding patient experiences and acting with patients to develop practical improvements and a more coproduced healthcare system.
\end{abstract}

\title{
Keywords
}

Patient experience, patient involvement, coproduction, person-centeredness, interactions, culture, quality improvement, $\mathrm{HIV} / \mathrm{AIDS}$

\section{Introduction}

There is growing recognition that patients should play a central role in defining, assessing, and improving healthcare quality, thereby enhancing patient experiences. To achieve this, healthcare organizations hope to align healthcare services with patients' identified values, preferences, and experiences. ${ }^{1}$ Increasingly, patients are also asked to engage in efforts to improve quality. ${ }^{2}$ These twin goals - to make healthcare more 'patient-centered' and to achieve it by encouraging 'patient involvement' in quality of care discussions and activities - are ambitious, and their realization challenges healthcare organizations for several reasons. When ascertaining patient-centered information, structured improvement efforts largely apply measurement-driven quality improvement techniques to quantify quality of care indicators or deploy surveys with pre-defined values. While measures are important when they are well established and comprehended by patients, they likely fail to capture patients' full experiences of care, which are harder to quantify. Patients often prefer to describe quality of care using personal stories about their experiences, to share the depth, texture, change over time, and the specific contexts in which features of quality are important to them. ${ }^{3,4}$ In terms of involvement, patients are increasingly invited to join advisory and quality improvement committees, or offer teach-back to staff about their experiences. ${ }^{5}$ However, patients may have limited quantitative skills to partake in typical quality improvement activities, and their representation is described as at times tokenistic, reproducing rather than challenging power dynamics and hierarchies between patients, providers, and administrators. Organizations may be wary sharing data with patients fearing exposure of service problems. ${ }^{6}$ Further, more involved patients may

Patient Experience Journal, Volume 8, Issue 2 - Special Issue: The Impact of Inequity \& Health Disparities on the Human Experience (C) The Author(s), 2021. Published in association with The Beryl Institute.

Downloaded from www.pxjournal.org 
fail to represent the diversity of views and experiences of patients receiving services, $7,8,9$

In this paper, we will describe the Healthcare Stories Project (HCSP), which aims to foster both patientcentered and patient-involved healthcare within healthcare organizations. HCSP was a demonstration project of the New York State Department of Health AIDS Institute Quality of Care (QOC) Program, committed to create patient involvement in health care organizations' quality programs by supporting the development of "venue[s] [for consumers] to identify improvement concerns and integrate [them] into a process to find solutions and develop improvement strategies." 10,11 In the first part of the paper, we briefly review patient involvement approaches informing HCSP and describe HCSP's approach, aims, and activities. The second part describes the HCSP rollout in New York State HIV outpatient healthcare delivery settings, and a qualitative process evaluation of a set of programs that adopted HCSP activities. The evaluation sought to understand if and how HCSP implementation supported the development of patient-centered care insights and increased patient involvement in quality activities within clinical settings. Findings can support scaling HCSP to more diverse settings.

\section{Building the Healthcare Stories Project (HCSP)}

\section{Existing Approaches}

Two approaches to advance patient-centered and patientinvolved healthcare upon which the HCSP draws are 'Experience-Based Co-Design' (EBCD) and 'coproduction theory.' EBCD is a method to develop collaborations between patients and providers to elicit and understand patients' experiences throughout healthcare delivery encounters and design or improve services to increase positive feelings and experiences in care ${ }^{12}$ Evaluations of EBCD across disease areas showed patients and staff building stronger and more trusting relationships, increasing understandings of one another's perspectives, getting useful feedback, and making meaningful service changes..$^{13}$ Coproduction is a theoretical approach with origins in the 1970s, and in recent years it has gained prominence in healthcare. Coproduction conceptualizes how patients and professionals co-create healthcare delivery processes and outcomes, whether intentionally or through natural actions taking place in the process of service delivery, including within healthcare settings. ${ }^{14,15}$ Coproduction in its basic form occurs within the patientprovider interaction as each actor bidirectionally shapes and is shaped by one another, with implications at the systems level. For example, a primary care provider issues a referral, and the patient makes the follow-up appointment; if they delay in scheduling the appointment, there may be significant personal health outcomes but also resulting consequential effects on the system which may ultimately treat them with more intensive resources. Or, if the patient schedules the appointment but does not show up, a different system impact results due to staff effort expended around rescheduling and contingency planning. ${ }^{16}$ In this way, healthcare is not done 'to' patients, but should be understood and appreciated as being done 'with' them. ${ }^{12}$

The coproduction dynamic and impact on the system is influenced by organizational, structural, and other factors; the longstanding relationships patients have with providers, such as in a chronic care setting like an HIV clinic, may elicit specific interactions that are less likely to occur in acute care settings, for example in encounters for surgical procedures. In such time-limited settings, there will be different coproduction dynamics but no matter a settings' baseline, coproduction can be encouraged and its value amplified by supporting clinic actors to recognize what coproduction is and how it works, and then by harnessing it through the involvement of patients in planning, delivery, and assessments. ${ }^{13}$ Such efforts build and transform systems by seeding opportunities to combine provider and patient views, experiences, actions and desires towards making a system that works better for everyone. ${ }^{17}$ Batalden et al. describe the skills patients and providers need to participate in shared work, particularly at the systems level, as: readiness and curiosity to engage, listening, willingness to learn new skills (e.g., data analysis), and building trust to openly share information and experiences. ${ }^{15}$ With the attainment of mutually learned skills and partnerships, better processes of care and associated health outcomes can be collectively achieved. ${ }^{18-19}$

\section{The Healthcare Stories Project (HCSP) Framework}

HCSP is designed as a platform of clinic-based activities to foster the identification of patient-centered care and increase patient involvement in quality of care activities within clinical settings with the goal of transforming the system of care to be more patient-centered (Figure 1). HCSP is informed by EBCD, coproduction theory, and findings from a formative ethnographic study conducted by the paper's authors that identified naturally occurring coproduction between patients and providers in HIV clinical settings. ${ }^{4,20}$ Specifically HCSP activities aim to: 1 ) Capture how patients define and experience 'quality of care' in the clinic; 2) Engage patients and providers as equal partners in understanding and improving the quality of care; and through partnerships 3) Support the building of a coproduced healthcare system.

HCSP comprises three activities, each paired to an anchor poster introducing staff and patients to the activity and illustrating the specific activity product to be generated (Figure 2). The activities are designed to be feasible and engaging and feed into achieving the overarching HCSP aims. Each activity can be undertaken individually, but 
Figure 1. Healthcare Stories Project Overview

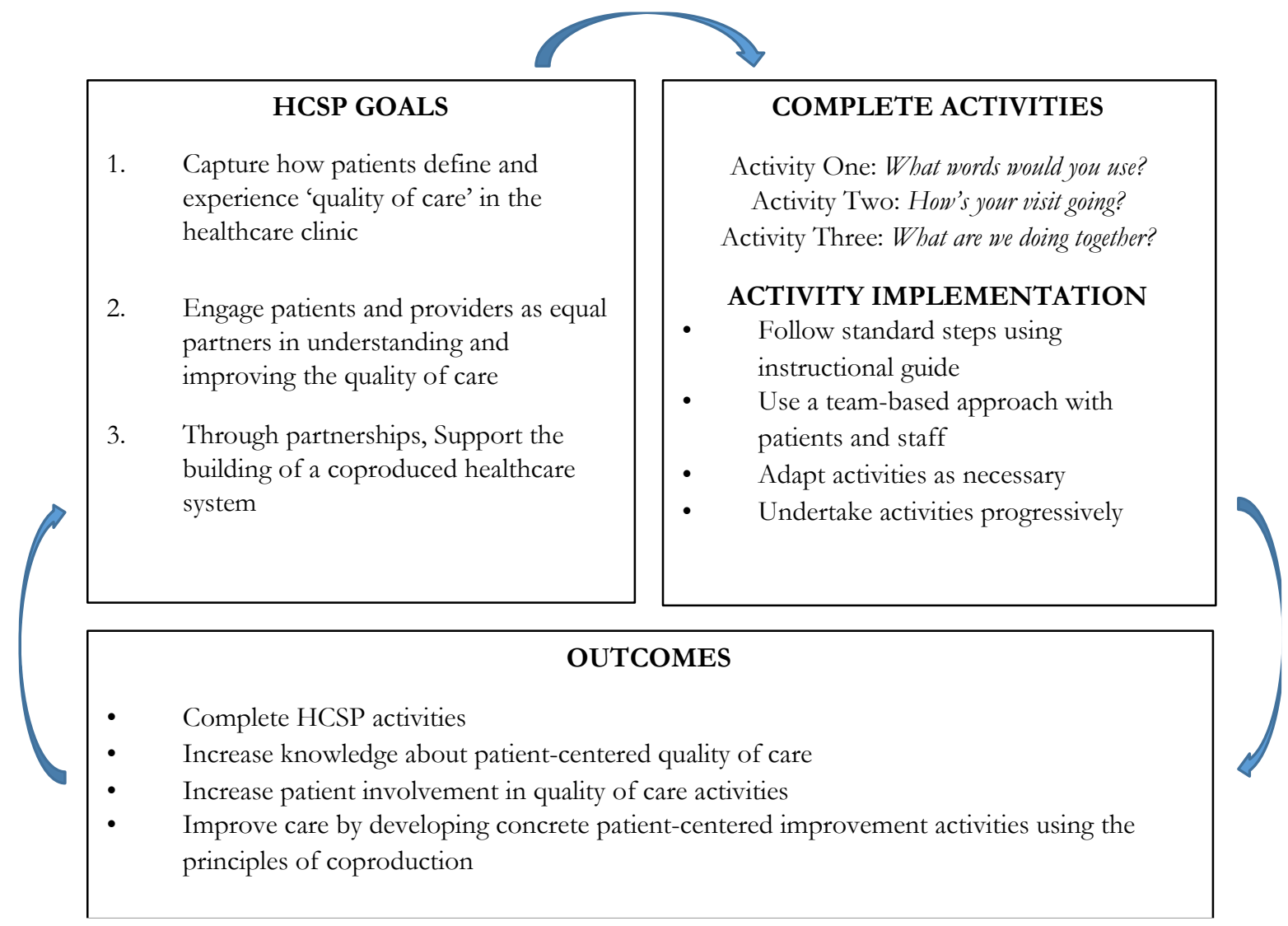

Figure 2. HCSP Three Activity Anchor Posters

Each poster is an illustration of the activity product completed by the health clinic

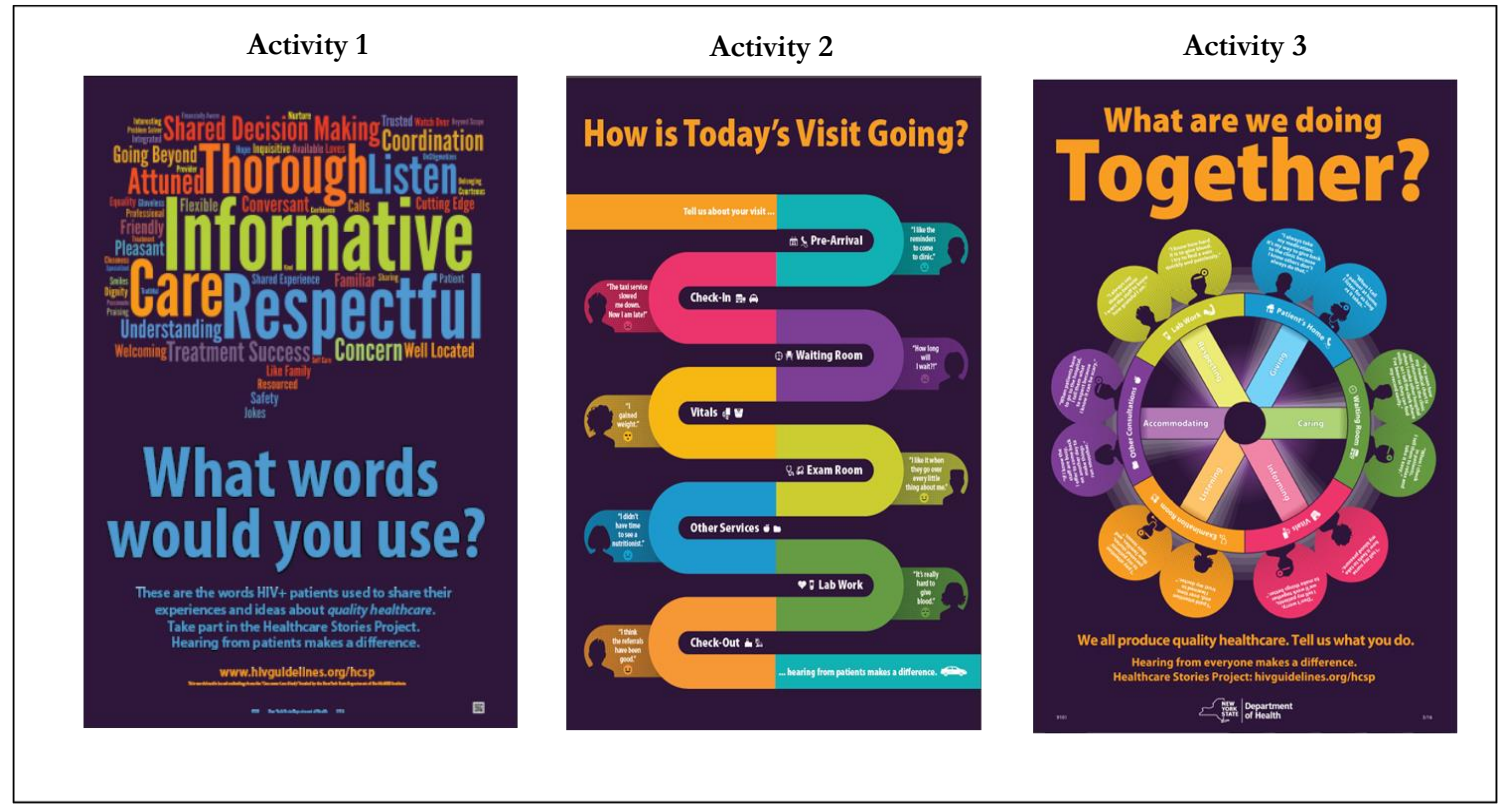


HCSP is designed to have the greatest effect on clinical change when the activities are implemented in succession, because skills and relationships may take time to build. ${ }^{14}$

Activity One, What words would you use? establishes awareness of patient-centered quality of care through the activity product of building a clinic-level, patient-defined word cloud along with stories that explain why these words are important to healthcare experiences. This word cloud serves as the beginning of building a shared understanding and culture of quality of care inclusive of patients' perspectives.

Activity Two, 'How's your visit going?' identifies patients' meaningful care experiences at specific service points in a healthcare visit. Borrowing from EBCD and patient journey mapping, ${ }^{21}$ patients describe with words and images their 'touch points': powerful emotional (positive or negative) feelings generated at specific service points. From this mapping, the clinic creates not a single patient visit journey like some mapping efforts, but an "Ideal Visit Map" based on aggregate experiences across patients that indicate how and where to achieve positive touch point experiences, and in turn to generate improvement projects to achieve them. Here, the healthcare organization moves from ideal concepts to applying them in the context of service delivery and in line with ideal experiences sought by patients.

Activity Three, 'What are we doing together?' is most directly underpinned by coproduction principles, set up to explore how patients and staff together produce or make healthcare services. The purpose is to identify specifically how and where coproduction occurs, to then strengthen and harness it and improve quality of care. For example, in the anchor poster, an observed example drawn from the formative study ${ }^{18}$ is how in the waiting room a front-line administrator reassures a patient that the doctor will see her shortly, while the patient practices patience and communicates patience to others who are also waiting to follow procedures and encourage calm. Activity Three solicits information from both patients and staff about what they 'do' in the healthcare delivery environment at each encounter point and synthesizes these combined efforts in a 'coproduction wheel' product, to inform while generating systems strengthening ideas.

Activity steps are standardized: 1) Assemble an HCSP team of patients and staff representing different disciplines to champion and implement the activity; 2) Display an anchor activity poster showing a sample activity product as a 'call to action' to participate (Figure 2); 3) Collect information from patients (and staff) using HCSPdesigned forms; 4) Analyze responses using HCSPdesigned worksheets; 5) Create the clinic-specific activity product and display it in the clinic; and 6) Share the activity with the wider clinical community, and develop one or several quality-related activities based on findings. A detailed step-by-step instructional guide including forms and worksheets accompanies each activity and delineates appropriate adaptations to maintain fidelity while allowing for site-level adaptations and tailoring. To be consistent with guidance on features associated with successful spread of innovations to staff in healthcare settings, activities are observable throughout implementation, and are as compatible as possible with (while developing) existing organizational practices. ${ }^{22}$

The activities are designed to achieve specific outcomes while also being mutually re-enforcing and amplifying. Each activity builds, and builds upon, one another. By the time of Activity Three, clinics should be equipped to improve quality of care through a patient-centered lens that strengthens and harnesses the potential of coproduction.

\section{HCSP Implementation in New York State}

AIDS Institute staff mailed HCSP Activities One and Two to 165 discrete HIV health organizations participating in the AIDS Institute QOC Program in March 2014 and December 2014, respectively. Staff mailed Activity Three in June 2016 to organizations known to have implemented Activities One or Two. All activities and supplemental materials were downloadable. ${ }^{23}$

To support adoption of HCSP activities, AIDS Institute staff and consultants (2-3 at any one time) promoted HCSP by presenting it meetings around New York State; making phone calls and in-person visits to organizations; coordinating with AIDS Institute quality coaches to promote engagement by organizations; and sending 'dear colleague' style letters from the AIDS Institute's Medical Director. To facilitate implementation, HCSP planners provided informational webinars and check-ins, an online platform for organizations to upload project results and communicate, and as-needed technical assistance.

\section{HCSP Demonstration Process Evaluation}

\section{Methods}

Data Collection

HCSP Project staff conducted a process evaluation with organizations known to implement the activities, representing hospital-based HIV specialty centers and community-based health centers (CHCs) of different sizes and from across New York State (Table 1). HL and MB who had not designed HCSP conducted semi-structured interviews after being trained by ABL, an expert in qualitative research. They conducted interviews with one staff member per organization $(n=12)$ after implementing Activity One between November 2014 and June 2015. A second round of 10 interviews was conducted after rolling out Activities Two and Three, and a reasonable period of time for implementation passed, between April and June 
Table 1. Healthcare Stories Project Participation Table

\begin{tabular}{|c|c|c|c|}
\hline & $\begin{array}{l}\text { Activity One } \\
\quad(n=12)\end{array}$ & $\begin{array}{l}\text { Activity Two } \\
\quad(\mathrm{n}=10)\end{array}$ & $\begin{array}{l}\text { Activity Three } \\
\quad(n=4)\end{array}$ \\
\hline $\begin{array}{l}\text { Designated AIDS Center } \\
\text { HIV Patient Census }\end{array}$ & $4 / 12$ & $4 / 10$ & $1 / 4$ \\
\hline Average & 995 & 1044 & 1550 \\
\hline Range & $280-1400$ & $225-1550$ & $n / a$ \\
\hline Median & 1150 & 1225 & $n / a$ \\
\hline Aggregate & 3980 & 4,175 & 1550 \\
\hline \begin{tabular}{|} 
Community Health Center \\
HIV Patient Census
\end{tabular} & $8 / 12$ & $6 / 10$ & $3 / 4$ \\
\hline Average & 539 & 838 & 617 \\
\hline Range & $100-990$ & $190-1378$ & $190-1177$ \\
\hline Median & 585 & 900 & 485 \\
\hline Aggregate & 4310 & 5,030 & 1852 \\
\hline
\end{tabular}

2017. Interviews took place with staff involved in HCSP (program directors, quality managers) though one clinic volunteer was also interviewed (Activity Two).

Interviewers used a semi-structured topic guide covering views of HCSP, completion, feasibility and process of implementation of activities, perceived achievements and challenges undertaking activities, and perceived changes or outcomes as a result of activities. Interviews lasted between 60-90 minutes. Interviewers recorded responses to the questions in the topic guide. In addition to interview data, staff gathered activity products from participating organizations, and took notes during check-in meetings. The HCSP evaluation protocol was submitted to the NYS Department of Health Institutional Review Board and received exemption status on June 9, 2014.

\section{Analysis}

$\mathrm{ABL}$ and $\mathrm{FC}$ analyzed interview summaries and program materials using a framework model to construct codes based on pre-conceived categories from the topic guide, as well as a set of iterated emergent codes and themes. ${ }^{24}$ The analysis focused first on the feasibility of completing each activity and then moved to implementation processes, and if and how the process produced knowledge about and enhanced patient involvement in quality of care. All members of the research team discussed and refined findings interpretation.

\section{Results}

\section{Activity Completion}

Three HCSP activities were implemented in succession by $36 \%$ (4/11) of participating organizations; $82 \%(9 / 11)$ of the organizations completing Activity One implemented Activity Two, and of those, almost half (4/9) went on to Activity Three. Several providers noted that repeating activity steps helped them feel capable of tackling the next activity. Interviewees called the activities 'creative' and 'fun' and contrasted with the 'usual improvement work,' which facilitated their willingness to continue the activities. These factors helped some organizations surmount barriers to implementation (competing priorities, staff turnover) mentioned ubiquitously across interviewees. Table 2 displays activity completion rates. 92\% (11/12) of interviewees said their organizations completed Activity One, producing and disseminating the word cloud. Over 850 patients participated, with an average of 72 and a median of 55 per organization. $60 \%(6 / 10)$ of the organizations completed Activity Two; 647 patients participated, or the equivalent of 80 per organization with a median of $50.25 \%(1 / 4)$ had started Activity Three and completed it, with the other three planning to finish; at the time of the interview 68 patients and 75 staff participated. Organizations made adaptations to the instruction guides to enable completion, including lengthening the timeline from 16 weeks as prescribed (contending with limited resources, competing priorities, and staff turnover), simplifying the information collection forms for patients with lower literacy levels, and translating forms to include non-English speaking patients. While designed to be implemented with HIV patients, the majority implemented Activity Two and Three with a broader patient population.

\section{Implementation Processes}

1. Enhanced Patient Roles and Groups Involved in Organization Activities

Many clinics opted to embed the HCSP activities into existing Consumer Advisory Boards (CABs) or Quality Committees. The $\mathrm{CAB}$ is a patient group designed to function in a consultative capacity to improve organization practice. The Quality Committee reviews performance data and develops mechanisms of improvement. Quality Committees may (but often do not) include patient representatives. CABs became central to HCSP activity planning and implementation at many organizations; $80 \%$ of Activity Two and 100\% of Activity Three interviewees reported CAB 
Table 2. Implementation Completion Details

\begin{tabular}{|c|c|c|c|}
\hline & $\begin{array}{c}\text { *Activity One } \\
(\mathrm{n}=12)\end{array}$ & $\begin{array}{c}\text { ^Activity Two } \\
(\mathrm{n}=10)\end{array}$ & $\begin{array}{l}\text { Activity Three } \\
(\mathrm{n}=4)\end{array}$ \\
\hline Activity (including Product) Fully Completed by & $92 \%(11 / 12)$ & $60 \%(6 / 10)$ & $25 \%(1 / 4)$ \\
\hline Interview $(\mathrm{Y})$ & Word Cloud & Ideal Visit Map & Coproduction Wheel \\
\hline Planning to Complete $(\mathrm{Y})$ & Not asked & $50 \%(2 / 4)[80 \%$ total $]$ & $100 \%(3 / 3)[100 \%$ total $]$ \\
\hline Staff Involved $(\mathrm{Y})$ & $100 \%(12)$ & $100 \%(10 / 10)$ & $100 \%(4 / 4)$ \\
\hline Consumer Advisory Board Involvement (Y) & Not asked & $80 \%(8 / 10)$ & $100 \%(4 / 4)$ \\
\hline \# Patient Participants & & & (out of 2) \\
\hline Average & 72 & 60 & 34 \\
\hline Range & $15-200$ & $10-174$ & $25-43$ \\
\hline Median & 55 & 53 & 34 \\
\hline Aggregate & 860 & 517 & 68 \\
\hline Includes HIV $+\&$ Other Patients $(Y)$ & Not asked & $80 \%(8 / 10)$ & $100 \%(4 / 4)$ \\
\hline \# Staff Surveyed & $\mathrm{n} / \mathrm{a}$ & $\mathrm{n} / \mathrm{a}$ & 75 \\
\hline
\end{tabular}

involvement (the question was not asked for Activity One). Interviewees said HCSP supported CAB growth. At one site, a newly-formed CAB made the HCSP Activity One its first formal undertaking, conferring a sense of purpose and accomplishment by, as one interviewee said, "seeing their voices come together to create a beautiful picture." Another interviewee said the CAB influenced clinic-wide improvements for the first time by founding a support group based on activity results. As the $\mathrm{CAB}$ gained prominence, interviewees reported expanding $\mathrm{CAB}$ attendance, with more individuals expressing interest in leadership roles.

By making activities for and executed by patients, HCSP became a vehicle to build patients' "professional skills" in quality of care. This included familiarization with systematic analysis techniques by helping to identify patterns and themes and translating findings into project ideas. Patients also led in creating visualized products; at one clinic, patients went beyond HCSP guidance to make a computer-generated word cloud by designing an image of a home, with positive words filling the interior space and negative ones puffed out as smoke from a chimney (Figure 3). Patients may have pre-existing artistic skills, but HCSP channeled them to communicate unique, patientcentered insights which they used to design the activity products. Another organization described how HCSP offered an opportunity to develop patients' presentation skills by, for example, co-presenting the activity at their annual clinical meeting.

\section{Greater Involvement of All Staff in Patient- Centered Quality of Care}

In addition to ramping up and focusing $\mathrm{CAB}$ involvement, interviewees said that front-line staff (from clerks to Patient Care Assistants to nurses) not typically included in quality management activities, engaged in quality of care conversations and improvement for the first time through HCSP. Translating and handing out forms, serving on the

Figure 3: Clinic Activity One Word Cloud Example

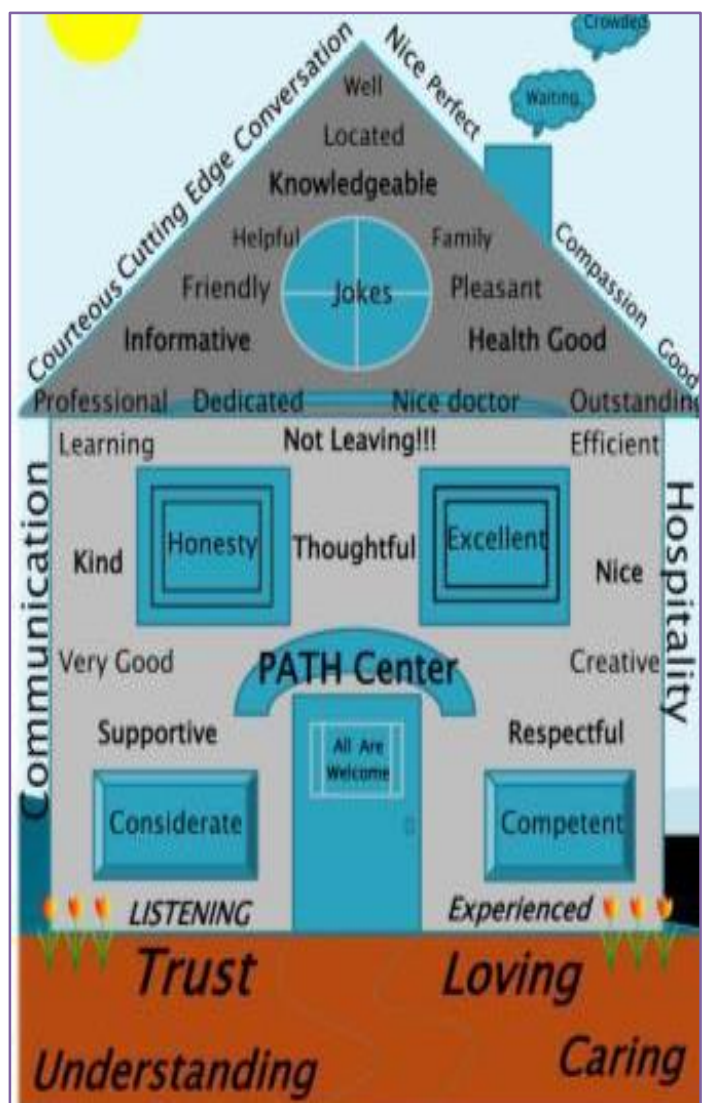


HCSP team, and presenting findings at monthly staff meetings provided opportunities to engage in quality of care discussions by articulating patients' needs and concerns. The visual nature of the activities was identified as an important cue for staff to become cognizant of the organization's concerns about quality of care and the importance of everyone taking part to define its meaning and action. Because many of the ideas shared by patients were of a positive nature, the activity products (i.e., word clouds and the ideal visit maps) made staff feel proud of the job they were doing. One organization's CAB created thank you cards (described below) and distributed them to staff in a public show of praise, which in turn was felt to boost staff morale. Providers noted the positive feedback served as a welcome antidote to the daily pressures of delivering healthcare and prompted a positive feedback loop for staff to go to greater lengths to help patients feel better. Interviewees praised the HCSP for helping the organization develop a patient-centered clinical identity, constituted by a community of patients and staff.

\section{Knowledge Gained and Ideas Generated to Improve Patient Experiences and Patient-Centered Quality of Care}

Interviewees expressed appreciation that activities were systematic and pattern-seeking, and therefore trustworthy and complementary with other gathered information like patient satisfaction surveys, and quality of care indicators. They felt that the resulting products showcased overall patient-centered quality of care values in visually meaningful ways. The activities opened up different types of ideas; Activity One yielded broader concepts and improvement areas, while Activity Two led to targeted insights and point of service improvements. The clinic that completed Activity Three felt it was a useful heuristic to build organizational understanding of concepts underpinning coproduction.

Across activities, one noted finding was how HCSP heightened staff awareness about the emotional experiences of patients coming into the clinic. One interviewee described how HCSP Activity One displayed the influence of stigma and discrimination on patient perceptions of care encounters. As a result, the organization instituted a sensitivity training for new staff to build a more patient-centered environment. Other organizations echoed that the activities helped them appreciate that patients feel vulnerable coming to the clinic and underscored the importance of peer programs to offer a "friendly face" and a "safe space" in the clinic. One interviewee said that her clinic developed a new patient support group to bolster this patient-centered sentiment. HCSP also surfaced emotions patients felt during what for staff were ordinary service elements. Through Activity Two, organized around positive and negative touch points, patients conveyed discomfort related to particular clinical tasks such as being weighed, taking a mental health assessment, and having blood drawn. These ordinary tasks were in fact moments in which patients felt particularly vulnerable. Such tasks were charged with further unhappiness while they waited for them to happen. Wait times repeatedly arose as a vexing issue, and as a result several organizations described strategies to reduce them, or instituting soft touches (e.g., check-ins, conveying approximate wait times) for patients at opportune moments.

HCSP led one organization to take the finding of discomfort waiting for bloodwork and conducted a followup analysis to measure wait times at different service points. The team learned that the wait to take blood was no longer compared to other points in which patients waited; the difference was how patients felt waiting at this point. The exercise revealed that the context of waiting, and the service one waits for, matters. The organization could then target patient support at this particularly challenging moment.

Based on the positive finding that the majority of patients felt gratitude to clinical staff and the care they received, one organization put together a 'thank you' project; this consisted of patients making and handing out thank you cards to front-line staff depicting their word cloud, along with 'Extra' chewing gum.

While most interviewed expressed positive sentiments about HCSP, two interviewees said the information generated during Activity Two confirmed what was "already known," with particular reference to the problem of wait times. These interviews represented organizations that did not involve CABs or patient volunteers in implementing the activities. While one interviewee said no activities resulted, the other still developed a feedback mechanism to address concerns at point of service based on activity results.

\section{Discussion}

Findings show that HCSP activities created a meaningful opportunity for patients to inform quality of care and participate in quality-related activities within their clinics. Insights gathered from patients ranged from broad sentiments (i.e., their values and the culture of care they sought) in Activity One, to point of service experiences in Activity Two, to mechanisms of service delivery in Activity Three. Through the analysis process, insights were contextualized and rendered more meaningful to (for example) reveal the power of stigma and discrimination in shaping care experiences, and the specific feelings that arise around certain services (mental health assessments, blood draws), and within particular contexts (waiting) compared to others. These kinds of experiences and 
concerns were systematically captured but were deeper than feedback generated through typical solicitation techniques like satisfaction surveys. The visual and interactive nature of the activities supported staff members to better recognize patients' ideas, experiences and feelings about quality of care, and to generate well-aligned concrete and practical organizational improvement ideas.

Undertaking activities also strengthened meaningful patient involvement in quality of care processes and structures and fostered patient-staff interactions. HCSP afforded a further opportunity to diffuse quality of care planning and implementation across front-line staff thought to not typically be involved. Each activity forged shared purpose, and as a result built both social and technical skills. By finding an implementation home through the CAB, HCSP was further leveraged to enhance the purpose of and expanded membership in a preestablished patient advocacy structure. On display were several of the mechanisms Palmer et al describe as leading to meaningful changes in coproduction processes, including: the building of recognition, dialogue, cooperation, creative attainment, enactment, accountability and mobilization. ${ }^{25}$ These moves enhanced the potential for patient involvement to transform from consultation to true partnership. ${ }^{26}$

Organizations that found HCSP to be less successful tended to frame activities as satisfaction surveys, with responses considered too positive or unsurprising. Studies have found shortcomings with satisfaction surveys, and so if activities were interpreted by staff in this way, they may not have found a meaningful fit within the organization. ${ }^{27}$ Organizations with a dimmer view of HCSP also seemed to downplay the role of the $\mathrm{CAB}$ or patients taking part in implementing the activity, while those organizations engaging their CABs seemed to have a deeper experience. This suggests that some degree of pre-existing recognition of patients' values may better leverage HCSP, compared to organizations that are hesitant about their usefulness.

The underpinning coproduction paradigm driving Activity Three was the most challenging to convey and apply through the activity. For the single organization that did complete it by the interview, the activity helped staff become more aware of the coproduction concept, and how patients have a role to play in clinical services. On one hand, this leads us to question whether Activity Three needs modification given its potential complexity, and if more work is needed to better understand the barriers for organizations to explicitly 'think' and 'do' coproduction. On the other hand, a coproduction ethos suffused each activity by fostering a shared understanding of and set of activities to work together. As a result, the HCSP goal of enhancing coproduced healthcare seeded opportunities for patients and providers to work together on equal footing, to in fact 'do' and enhance coproduction through each
HCSP activity. The amplifying effect of coproduction was graphically illustrated by the Activity One-inspired thank you card project to bolster staff morale, truly 'blurring the boundaries' between traditional and professional roles. ${ }^{5}$ Further, by using HCSP to assign the CAB greater purpose, patient resources ramped up ${ }^{28}$ to become more explicitly geared to co-creating clinical improvements, thereby offering another opportunity to support patients to become 'movers and doers' in the clinical environment. A future version of HCSP Activity Three might build upon the Activity One and Two generated ideas to strengthen patient-centered organizational processes embedded with patient involvement.

\section{Limitations}

There are several limitations to these findings. The number of tracked organizations is relatively small and may not be representative of HIV clinics across New York or beyond. However, this limitation was mediated by sampling from diverse geographic regions and organization types. Participating organizations serve diverse panels in terms of race, ethnicity, and gender, but it is unknown if those who participated are as diverse. Similarly, another limitation is the potential exclusion of patients unwilling or unable (e.g., due to literacy or language issues) to partake in HCSP activities. While organizations adapted the activity to foster inclusion and engaged large number of their patient panels, they may not have surmounted obstacles to engage the hardest to reach. Finally, interviews were conducted with staff; in the future, gathering 'user' experiences from patients will yield additional insights into the benefits and drawbacks related to the HCSP process.

Another limitation lies in the lack of formal metrics to evaluate the degree of participation, health, or organizational outcomes as a result of HCSP. Findings might be strengthened by using a quality improvement or implementation science approach to measure the types of implemented activities as a result of HCSP, and their outcomes on improving clinical, organizational, or other defined metrics including engagement in care. HCSP was not evaluated by longer-term outcomes by participants, sustainability of improvement initiatives, or organizationlevel changes. Further, while HCSP seemed to improve patient-centered and patient-involved care, the lack of measuring the intensity of that relationship does not allow us to describe the extent to which or the degree of partnership and its effects. By developing these measures and study designs in future, we will be better positioned to explore whether fostering a patient-involved and patientcentered environment has lasting benefits, as well as whether the engagement of some patients diffuses as gains for all.

Finally, HCSP should be used and evaluated outside of the HIV context to transfer learning, honed for decades with 
strong commitments to patient empowerment, advocacy and inclusion, to other patient populations. HCSP shows promising signs of wider applicability; the fact that many organizations included non-HIV patients in the activities demonstrates HCSP's potential reach and supports its adaptability into other healthcare contexts. Initial dissemination might be to disease communities with histories of similar advocacy such as for some cancers, muscular dystrophy, and cystic fibrosis. ${ }^{11}$ Other healthcare contexts, such as primary care settings where routine chronic disease management occurs but where there are no pre-existing advocacy groups may also take up HCSP given that our evaluation showed HCSP to influence organizational norms and provider views to become more open to patient involvement overall. Another context of HCSP implementation that might be fruitful to explore would be settings primed to work on service redesign or similar activities, and open to focused activities like HCSP to organize their process. Research will be needed when applying HCSP in these varied settings to support knowledge building around coproduction, and its influencing factors. If this model is shown to be effective in broader contexts, ultimately HCSP should be integrated it into a diversity of healthcare settings.

\section{Conclusion}

HCSP is a platform to build meaningful patient involvement in quality of care using principles of experience-based design and coproduction. As the demonstration project showed, HCSP enabled understandings of quality by both patients and staff and generated more inclusion of patients and front-line workers in quality processes and improvement initiatives. HCSP looks to be a promising and innovative addition to the toolkit to build truly patient-centered and patientpartnered healthcare systems.

\section{Acknowledgements}

We would like to acknowledge each of the HCSP participating clinics' patients and staff who took part in the activities and the evaluation. We would particularly like to thank the Brooklyn Hospital PATH Center for graciously providing their word cloud. We would also like to deeply thank Michele Canfield for her excellent design work on the HCSP materials, and the NYS Department of Health statewide Consumer Advisory Committee for their active engagement with this initiative. This paper is dedicated to the memory of Humberto Cruz, former AIDS Institute Director, and champion of advancing coproduction with the PLWHA community.

\section{References}

1. Institute of Medicine. Crossing the Quality Chasm: A New Health System for the 21st Century. Washington, DC: The National Academies Press; 2000. doi: $10.17226 / 10027$.

2. Wolf J. Patient experience: the new heart of healthcare leadership. Front Heal Serv Man. 2017;33(3), 3-16. doi:10.1097/HAP.0000000000000002.

3. Bate SP, Robert G. Bringing user experience to healthcare improvement: the concepts, methods and practices of experience-based design. Oxford: Radcliffe Publishing; 2007.

4. Baim-Lance A, Tietz D, Schlefer M, Agins B. Health Care User Perspectives on Constructing, Contextualizing, and Co-Producing "Quality of Care." Qualitative Health Research. 2015; 26(2): 252-263. doi:10.1177/1049732315569736

5. Renedo, A, Marston C. Spaces for citizen involvement in healthcare: an ethnographic study. Sociology. 2015; 49(3): 488-504. doi:10.1177/0038038514544208.

6. Batalden M, Batalden P, Margolis P et al. Coproduction of healthcare service. BMJ Qual Saf, 2015; 0: 1-9. doi:10.1136/bmiqs-2015-004315.

7. Boote J, Telford R, Cooper C. Consumer involvement in health research: a review and research agenda. Health Policy. 2002; 61(2): 213-236. doi: 10.1016/S01688510(01)00214-7.

8. deFreitas C, Martin G. Inclusive public participation in health: Policy, practice and theoretical contributions to promote the involvement of marginalised groups in healthcare. Social Science and Medicine. 2015; 135: 31 39. doi:10.1016/j.socscimed.2015.04.019.

9. Pomey, M. Patient partnership in quality improvement of healthcare services: patients' inputs and challenges faced. Patient Experience Journal. 2015; 2(1): 29-42. doi:10.35680/2372-0247.1064.

10. New York State Department of Health AIDS Institute. The HIV Quality of Care Program.

https://www.health.ny.gov/diseases/aids/general/abo ut/quality.htm accessed November 30, 2020.

11. Coren F, Brown M, Ikeda D, et al. Beyond tokenism in quality management policy and programming: moving from participation to meaningful involvement of people with HIV in New York State. Int J Qual Health Care. 2021; 33(1). doi:10.1093/intqhe/mzab004.

12. Bate SP, Robert G. Experience-based design: from redesigning the system around the patient to codesigning services with the patient. Qual Saf Health Care, 2006; 15(5): 307-310.

13. Clark D, Jones F, Harris R, et al. What Outcomes are Associated with Developing and Implementing CoProduced Interventions in Acute Healthcare Settings? A Rapid Evidence Synthesis. BMJ Open 2017:7(7). doi:10.1136/qshc.2005.016527. 
14. Bovaird, T, Loeffler, E. The Role of Co-Production for Better Health and Wellbeing: why we need to change. In Loeffler E, Power G, Bovaird T, Hine-Hughes F, eds. Co-production of health and wellbeing in Scotland. Birmingham, UK: Governance International, 2013, p22.

15. Batalden M, Batalden P, Margolis P et al. Coproduction of healthcare service. BMJ Qual Saf, 2015:1-9. doi: 10.1136/bmjqs-2015-004315.

16. Loeffler E, Power G, Bovaird T, Hine-Hughes F, eds. Co-production of health and wellbeing in Scotland. Birmingham, UK: Governance International, 2013.

17. Needham C. Co-production: an emerging evidence base for adult social care transformation. SCIE Research Briefing 31. 2009. https://lx.iriss.org.uk/sites/default/files/resources/bri efing31.pdf. Accessed May 5, 2021.

18. Sabadosa K, Batalden P. The Interdependent roles of patients, families, and professional in cystic fibrosis: a system for the coproduction of healthcare and its improvement. BMJ Qual Saf 2014;23:i90-i94. doi: 10.1136/bmjqs-2013-002782.

19. Ocloo J, Garfield S, Dawson S, et al. Exploring the theory, barriers and enablers for patient and public involvement across health, social care and patient safety: a protocol for a systematic review of reviews. BMJ Open 2017;7:e018426. doi:10.1136/bmjopen2017-018426.

20. Baim-Lance A, Tietz D, Lever H, et al. Every day and unavoidable coproduction: exploring patient participation in the delivery of healthcare services. Soc Heal Ill. 2019; 41(1):128-142. doi: 10.1111/14679566.12801.

21. McCarthy S, O'Raghallaigh P, Woodworth S. An integrated patient journey mapping tool for embedding quality in healthcare service reform. J Dec Sys. 2016;25(supp1):354-368. doi:10.1080/12460125.2016.1187394.

22. Greenhalgh, T. Robert G., MacFarlane F, et al. Diffusion of Innovations in Service Organizations: Systematic review and recommendations. Milbank Quarterly. 2004; 82(4):581-629. doi: 10.1111/j.0887378X.2004.00325.x.

23. Healthcare Stories Project Materials. New York State Quality of Care Program, Activities to Promote Consumer Involvement in Quality Improvement. https:/ / quality.aidsinstituteny.org/ConsumerInvolve/ ConsumerInvolve/Welcome. Accessed November.

24. Gale NK, Health G, Cameron E, Rashid S, Redwood S. Using the framework method for the analysis of qualitative data in multi-disciplinary health research. BMC Medical Research Methodology. 2013;13(117). doi: 10.1186/1471-2288-13-117.

25. Palmer VJ, et al. The Participatory Zeitgeist: an explanatory theoretical model of change in an era of coproduction and codesign in healthcare improvement.
Med Humanities. 2019;247-257. doi: 10.1136/medhum-2017-011398.

26. Carman K, Dardess P, Maurer M. Patient and family engagement: a framework for understanding the elements and developing interventions and policies. Heal Aff. 32(2). doi:10.1377/hlthaff.2012.1133.

27. Zastowny T, Stratman W, Adams E, Fox M. Patient satisfaction and experience with healthservices and quality of care. Quality Management in Health Care. 1995; 3(3):50-61. doi:10.1097/00019514-19950303000006.

28. Renedo A, Marston CA, Spyridonidis D, Barlow J. Patient and public involvement in healthcare quality improvement: how organizations can help patients and professionals to collaborate. J Pub Man Rev.2015;17(1). doi: 10.1080/14719037.2014.881535. 